\title{
Modification of Endoscopic Dacryocystorhinostomy in Chronic Dacyocystitis
}

DOI: $10.17691 / \mathrm{stm} 2019.11 .2 .14$

Received September 30, 2018

G.S. Bogdanov, MD, PhD, Jutor, Department of Eye Diseases';

R.A. Larin, Head of the 1st Otolaryngological Unit2;

S.y. Ryazantsev, MD, DSC, Professor, Deputy Director for Scientific Coordination Work with Regions ${ }^{3}$

1 Privolzhsky Research Medical University, 10/1 Minin and Pozharsky Square, Nizhny Novgorod, 603005, Russia;

2 Nizhny Novgorod Regional Clinical Hospital named after N.A. Semashko, 190 Rodionova St., Nizhny Novgorod,

603126, Russia;

3 Saint-Petersburg Institute of Ear, Nose, Throat, and Speech, Ministry of Health of the Russian Federation,

9 Bronnitskaya St., Saint Petersburg, 190013, Russia

The aim of the study was to assess the efficacy of endosurgical treatment of chronic dacryocystitis using modified endoscopic dacryocystorhinostomy by analyzing the short-term and remote results.

Materials and Methods. In the period from 2012 to 2018, 120 operations for chronic dacryocystitis, 87 of which were endoscopic endonasal dacryocystorhinostomias (EEDCR), have been performed in the Clinic of Eye Diseases and ENT Clinic at Nizhny Novgorod Regional Clinical Hospital named after N.A. Semashko. Chronic dacryocystitis was caused by stenosis of the nasolacrimal ducts. Women prevailed in this cohort of patients. The age of the patients ranged from 23 to 82 years. Of 87 patients undergone EEDCR, 45 had phlegmon of the lacrimal sac in the medical history.

The operations were performed under intubation anesthesia. Eight patients were subjected to nasal septum correction as a first stage of surgical treatment.

All patients were seen for dynamical followed-up visits 15, 30, 90, and 180 days after the surgical intervention with obligatory examination of the nasal cavity and flushing of the lacrimal passages.

Results. In 79 patients (90.8\%) patency of the lacrimal ducts was restored. In 16 individuals (18\%) there was recurrence of dacryocystitis at different terms ( 1 to 6 months) requiring repeated operations. The recurrent episodes were related to the specific anatomy of the nose cavity, in particular, due to a proximal location of the middle nasal concha to the junctionary. A complex examination of patients with dacryocystitis allows ophthalmologists or ENT specialists to avoid mistakes in establishing the diagnosis and reduce intraoperative and postoperative complications. A two-step operation technique (correction of the nasal septum curvature and dacryocystorhinostomia) makes it possible to improve visualization of the operative field, reduce the risk of recurrences, diminish anesthesiological load and stress for the patient. Preliminary diathermocoagulation of the nasal mucous membrane prior to flap dissection on the lateral wall reduces bleeding during the operation. Transcanalicular intraoperative illumination of the lacrimal sac projection enables surgeons to choose precise junctionary topography.

Conclusion. A modified EEDCR with transcanalicular illumination of the lacrimal sac and preliminary diathermocoagulation of the nasal mucous membrane in the lacrimal sac projection is an effective surgical treatment of dacryocystitis, while preoperative examination by the ENT specialist and computed tomography of the paranasal sinuses and ethmoidal labyrinth provide the opportunity to adequately plan the operation and decrease the recurrence rate.

Key words: dacryocystitis; endoscopic dacryocystorhinostomy.

\section{Introduction}

Among all patients with vision organ diseases, the share of those suffering from tear drainage disorders is $6-25 \%$, of this number $2.0-7.5 \%$ had chronic dacryocystitis [1]. Dacryocystitis in the general structure of eye pathology accompanied by the inflammatory process of the vertical part of the tear drainage system amounts to $2.0-7.6 \%$. The disease manifests more often in women at the age of $30-60$ years (7-8 times more often than in men) [1, 2]. This is explained by the anatomical structure of the tear drainage passages in females: a longer, narrow, and curved canal, high susceptibility to lumen stenosis and filling of its wall venous plexuses with blood during menstrual cycles [1, 2].

The most constant symptom of this disease is persistent lacrimation and sometimes purulent discharge. Excessive tearing and tear stasis result in vision blurring, reduced working capacity, limitations in profession selection determining medical and social importance of

Corresponding author: Georgy S. Bogdanov, e-mail: Hirurg1980@mail.ru 
this disease. Chronic purulent dacryocystitis is often the cause of suppurative corneal ulcer developing in case of insignificant epithelium injury near the lacrimal sac phlegmon which serves as an absolute contraindication to the planned operations on the eyeball.

The main cause of dacryocystitis is inflammation of the nasolacrimal duct, the initial localization of the inflammation being the nasal cavity and/or paranasal sinuses. Thus, etiologically and pathogenically grounded approach to the diagnosis and management of this illness must be based on a thorough examination of the ENT organs by otorhinolaryngologists and ophthalmologists in the form of an interdisciplinary dialogue.

If conservative therapy of chronic dacryocystitis proves to be ineffective, surgical treatment is performed. Endoscopic endonasal dacryocystorhinostomy (EEDCR) as a current method of operative treatment of dacryocystitis meets the principles of functionality and safety [1-4]. The method consists in formation of a stable communication between a lacrimal sac and nasal cavity, i.e. dacryocystorhinostoma.

The techniques implying an external approach (A. Toti, 1904) are of great historical importance however they can be used in unsuccessful repeated revision endonasal dacryocystorhinostomy. The founders of the endosurgical technique are J. West (1910), L. Polyak (1912), and D. Halle (1914). There are many modifications of endonasal dacryocystorhinostomy which were suggested by S.E. Stavraki (1924), V.O. Rudakov (1925), F.S. Bokstein (1929), and others. They differ in the way of forming the bone window and in the shape of the dissected flap of the mucous membrane on the lateral nasal wall. In Russia, methods of endosurgical treatment and their modifications were developed by G.Z. Piskunov, S.Z. Piskunov, V.S. Kozlov, and A.S. Lopatin (2006).

At present, recurrent dacryocystitis after ineffective operations, the necessity of lacrimal sac extirpation, immediate low traumatic intervention (in patients susceptible to tissue bleeding complicated by somatic status), frequently recurrent phlegmons of the cellular tissue surrounding the lacrimal sac especially after unsuccessful dacryocystorhinostomy serve as indications to EEDCR $[1,2,4,5]$.

One of the main principles of surgical treatment is prevention of stricture formation and scarring of the formed endonasal stoma, since these processes determine a high probability of dacryocystitis recurrence.

The aim of the study was to assess the efficacy of endosurgical treatment of chronic dacryocystitis using modified endoscopic dacryocystorhinostomy by analyzing the short-term and remote results.

\section{Materials and Methods}

In the period from 2012 to 2018, 120 operations for chronic dacryocystitis, 87 of which were EEDCR, have been performed in the Clinic of Eye Diseases and ENT Clinic at Nizhny Novgorod Regional Clinical Hospital named after N.A. Semashko. There were 79 (88\%) women and $11(12 \%)$ men. The age ranged from 23 to 82 years.

The study complied with the Declaration of Helsinki (2013) and was approved by the Ethics Committee of Privolzhsky Research Medical University. Written informed consent was obtained from each patient.

Chronic dacryocystitis was caused by stenosis of the nasolacrimal duct and in half of the patients lacrimal sac phlegmon was noted in their medical history. On admission to the inpatient department, patients presented complaints of persistent lacrimation and purulent discharge from the eyes.

On examination, excessive tearing along the margin of the inferior eyelid, a round soft skin protrusion under the internal eyelid ligament was observed. When pressing the lacrimal sac area, a flux of mucous and mucopurulent content was discharged from the lacrimal puncta.

A West fluorescein test (canalicular) and active lacrimal-nasal test were used with a diagnostic purpose. The canalicular test was positive in all patients whereas the lacrimal-nasal was negative. Passive lacrimal-nasal test was also conducted. When lacrimal ducts were washed, the fluid did not pass into the nose and flushed out through a free superior lacrimal punctum together with the sac content. At the same time, in the patients of the given group no stenosis of the lacrimal canaliculi was observed.

In a preoperative period, all patients underwent CT examination of the paranasal sinuses, rhinoscopy to diagnose nose septum curvature, polyps in the nasal passages, and other anatomic deviations and pathological conditions which, on the one hand, may contribute to the development of chronic dacryocystitis, and, on the other hand, essentially complicate endonasal operations and negatively influence the short-term and remote functional results. On the whole, about half of the patients were found to have some changes in the nasal cavity.

\section{Results}

After adequate preparation and additional examination, EEDCR was performed in 87 patients in order to create a straight junctionary between the lacrimal sac and nasal cavity. The operation was done under intubation anesthesia.

In 18 patients, correction of the curved nasal septum was carried out as a first step of the operation to facilitate the approach to the lacrimal sac and reduce the risk of postoperative stoma atresia. To access the lacrimal sac projection, the zone of the so-called tuber in the anterosuperior part of the nasal septum was chosen. This tactics is most suitable for the best visualization of the operative field in the middle nasal passage and 
reduction of recurrences in the postoperative period as a tightly adhering nasal septum in the projection of the formed junctionary worsens aerodynamics of the air stream and functioning of the mucociliar transport of the mucous membrane inevitably leading to adhesion formation and subsequent complete or partial obliteration of the bone window.

A $4 \mathrm{~mm}$ endoscope with 30 and 0-degree optics was used in our work.

Specific features of surgical intervention in our modification are as follows:

1. Before the dissection of a mucous flap on the lateral nasal wall, sparing target diathermocoagulation of the flap formation area was performed to reduce hemorrhage. An excessive bleeding in the operative field may significantly worsen visualization of the lacrimal sac and promote scarring in the postoperative period.

2. The most difficult task in this kind of intervention is exact localization of the lacrimal sac projection on the lateral wall of the nasal cavity. Inaccuracies at this stage may result in the formation of an oversized bone window increasing the volume of the surgical trauma and influencing mucous membrane functionality with all the consequences that this entails, or the bone window will not at all match the sac projection and incompetence of the stoma in the remote period is unavoidable.

The projection of the lacrimal sac to the lateral nasal wall was determined with the help of a rigid $23 \mathrm{G}$ optical fiber for vitreous surgery inserted into the inferior lacrimal canaliculum (see the Figure). A surgeon visualized the area of transillumination through the nasal mucous membrane on the side of the middle nasal passage which allowed the necessary incision projection to be determined. The lacrimal sac was illuminated till the

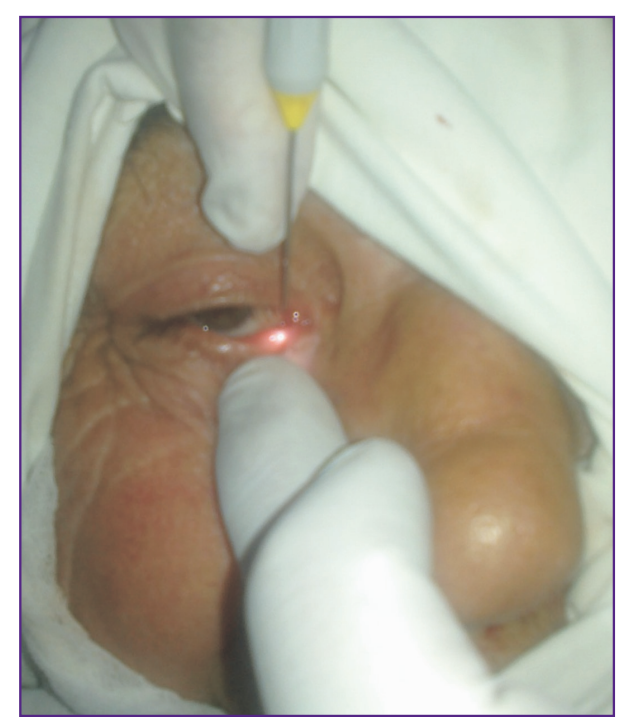

Intraoperative probing of the lacrimal canaliculi stage of bone window formation. Using a bur (we believe it to be more preferable than cutting forceps) with a 7-10 $\mathrm{mm}$ diameter in the projection of the lacrimal sac, a $10 \times 10 \mathrm{~mm}$ bone window was drilled in the bone using the optical fiber illumination as a guide. The cavity of the lacrimal sac was opened with a crescent knife. The lacrimal ducts were flushed till the appearance of fluid in the nasal cavity. A gauze turunda with Levomecol was inserted into the middle nasal passage with the proximal end introduced in the bone window area. The turunda was left in the nasal passage for 3 days. After its removal, the lacrimal ducts were flushed once a day for 3 days.

All patients underwent obligatory examination of the nasal cavity and flushing of the lacrimal ducts $15,30,90$, and 180 days after the operation.

The analysis of the results showed that in 79 cases $(90.8 \%)$ the patency of the lacrimal ducts was restored already in the early postoperative period. Absence of the functional result was observed in the patients with marked forms of hypertrophic rhinitis, chronic inflammation and edema in the nasal cavity, atypical location of the lacrimal sac, and in the group of patients with a persistently recurring course.

In the remote period, at different terms (1-6 months), the recurrence of dacryocystitis occurred in 16 of 87 patients $(18 \%)$ requiring repeated or revision operations. The recurrences were related to the specific anatomy of the nasal cavity and chronic inflammation of the mucous membrane.

An important and sometimes a leading cause of recurrence development is absence of interdisciplinary collaboration, correct routing of patients during postoperative treatment, follow-up and successive tactics of residential care.

\section{Conclusion}

The modified version of endoscopic dacryocystorhinostomy enables surgeons to improve functional results of surgical treatment.

A complex examination of patients with dacryocystitis by an ophthalmologist and ENT specialist provides the opportunity to minimize errors at establishing the diagnosis, reduces complication in the intraoperative and postoperative periods. The operative technique in the simultaneous format, i.e. correction of the endonasal structures and dacryocystorhinostomy, improves visualization of the operative field, diminishes the risk of recurrences, reduces anesthesiological load and stress to the patient. Preliminary diathermocoagulation of the nasal mucous membrane prior to flap dissection on the lateral wall reduces bleeding during the operation. Transcanalicular intraoperative transillumination of the lacrimal sac projection enables surgeons to choose precise formed junctionary topography.

Study funding. The work was not supported by any financial sources. 
Conflicts of interest. The authors have no conflicts of interest to declare.

\section{References}

1. Aznabayev M.T., Valiyeva G.N., Babushkin A.E. Failure reasons and prophylaxis of formed fistula cicatrization at dacryocystorhinostomy. Russkiy meditsinskiy zhurnal. Klinicheskaya oftal'mologiya 2006; 7(4): 171-174.

2. Krakhovetskiy N.N. Sravnitel'nyy analiz sposobov formirovaniya dakriostomy pri endoskopicheskoy endonazal'noy dakriotsistorinostomii. Avtoref. dis. ... kand. med. nauk [Comparative analysis of the methods of dacryostomy formation in endoscopic endonasal dacryocystorhinostomy. PhD Thesis]. Moscow; 2015.

3. Bobrov D.A., Zhukov S.K., Slezkina I.G. Application of the Ritleng intubation lacrimal system for the surgical treatment of combined lesions of lacrimal passages. Vestnik otorinolaringologii 2010; 2: 55-57.

4. Beloglazov V.G., At'kova E.L., Abdurakhmanov G.A., Krakhovetskii N.N. Prevention of ostial obstruction after microendoscopic endonasal dacryocystorhinostomy. Vestnik oftal'mologii 2013; 129(2): 19-22.

5. Yartsev V.D., Atkova E.L., Krakhovetskiy N.N. Modern technology of lacrimal passage restoration in dacryostenosis. Oftal'mohirurgia 2014; 2: 85-91. 